\title{
Compact expressions for spherically averaged position and momentum densities
}

\author{
Deborah L. Crittenden ${ }^{\text {a) }}$ and Yves A. Bernard \\ Research School of Chemistry, Australian National University, Canberra ACT 0200, Australia
}

(Received 2 June 2009; accepted 22 July 2009; published online 7 August 2009)

\begin{abstract}
Compact expressions for spherically averaged position and momentum density integrals are given in terms of spherical Bessel functions $\left(j_{n}\right)$ and modified spherical Bessel functions $\left(i_{n}\right)$, respectively. All integrals required for ab initio calculations involving $s, p, d$, and $f$-type Gaussian functions are tabulated, highlighting a neat isomorphism between position and momentum space formulae. Spherically averaged position and momentum densities are calculated for a set of molecules comprising the ten-electron isoelectronic series $\left(\mathrm{Ne}-\mathrm{CH}_{4}\right)$ and the eighteen-electron series $\left(\mathrm{Ar}-\mathrm{SiH}_{4}, \mathrm{~F}_{2}-\mathrm{C}_{2} \mathrm{H}_{6}\right)$. (C) 2009 American Institute of Physics. [DOI: 10.1063/1.3204011]
\end{abstract}

\section{INTRODUCTION}

Global properties of molecular systems are often independent of molecular anisotropy due to the rapid and often large-amplitude rotational component of classical thermal and quantum zero-point motion. In such cases, electronic and magnetic properties may be calculated directly as moments of the spherically averaged density of the system. For example, magnetic susceptibilities, ${ }^{1}$ nuclear magnetic shielding constants, ${ }^{2}$ and electric field gradients ${ }^{3}$ may all be obtained from nucleus-centered spherically averaged position space densities $\rho(r)$, while the spherically averaged momentum space density $\pi(p)$ can be exploited to find the electronic kinetic energy ${ }^{3}$ and extract experimentally observable $\mathrm{x}$ ray and electron scattering parameters. ${ }^{4,5}$ Therefore, it is useful to be able to accurately and efficiently generate spherically averaged position and momentum space densities.

Spherically averaged densities have a patchy history in the scientific literature. ${ }^{3,6-18}$ In their seminal 1994 paper on the topic, Wang and Smith ${ }^{3}$ presented a very general method for calculating spherically averaged densities and their moments based on a complicated expression involving coefficients with seven indices that are generated by four-term recurrence relations. Since then, simpler expressions for $\rho(r)$ were presented by Sarasola et al., ${ }^{8}$ while Thakkar and Sharma ${ }^{9}$ used spherical Bessel functions to simplify the integrals required to calculate $\pi(p)$. A number of subsequent studies have examined basis set, correlation, and solvation effects on radial position and momentum space densities. ${ }^{10-15}$

In the present work, we reproduce the momentum-space results of Thakkar and Sharma ${ }^{9}$ and show how an analogous approach can be applied to generate compact expressions for spherically averaged position space integrals. All developments presented here will be available in the next release of the Q-CHEM software package. ${ }^{19}$

\footnotetext{
${ }^{a)}$ Electronic mail: deborah@ @rsc.anu.edu.au.
}

\section{THEORY}

\section{A. Spherically averaged position space densities}

If the wave function of a molecular system is expanded in a basis of one-electron functions $\phi_{a}(\mathbf{r})$, its spherically averaged position density about the origin is given by

$$
\rho(r)=\sum_{a b} P_{a b} \int \phi_{a}(\mathbf{r}) \phi_{b}(\mathbf{r}) r^{2} \sin \theta d \theta d \phi,
$$

where $P_{a b}$ is the $(a, b)$ th element of the one-particle density matrix. If $\phi_{a}$ and $\phi_{b}$ are unnormalized $s$-type Gaussian basis functions centered at $\mathbf{A}_{0}$ and $\mathbf{B}_{0}$ with exponents $\alpha$ and $\beta$, the above integral can be found in closed form

$$
\begin{aligned}
{[s s]_{r} } & =\int \exp \left(-\alpha\left(\mathbf{r}-\mathbf{A}_{0}\right)^{2}-\beta\left(\mathbf{r}-\mathbf{B}_{0}\right)^{2}\right) r^{2} \sin \theta d \theta d \phi, \\
& =4 \pi r^{2} \exp \left(-\alpha\left(r^{2}+A_{0}^{2}\right)-\beta\left(r^{2}+B_{0}^{2}\right)\right) i_{0}\left(r P_{0}\right),
\end{aligned}
$$

where $\mathbf{P}_{0}=2 \alpha \mathbf{A}_{0}+2 \beta \mathbf{B}_{0}$ and $i_{0}(x)=x^{-1} \sinh x$ is a modified spherical Bessel function of the first kind. For spherically averaged position densities centered about an arbitrary origin C, a simple coordinate shift yields analogous integrals

$$
\begin{aligned}
{[s s]_{r} } & =\int \exp \left(-\alpha(\mathbf{r}-\mathbf{A})^{2}-\beta(\mathbf{r}-\mathbf{B})^{2}\right) r^{2} \sin \theta d \theta d \phi, \\
& =4 \pi r^{2} \exp \left(-\alpha\left(r^{2}+A^{2}\right)-\beta\left(r^{2}+B^{2}\right)\right) i_{0}(r P),
\end{aligned}
$$

where $\mathbf{A}=\mathbf{A}_{0}-\mathbf{C}, \mathbf{B}=\mathbf{B}_{0}-\mathbf{C}$, and $\mathbf{P}=2 \alpha \mathbf{A}+2 \beta \mathbf{B}$. $\mathbf{C}$ is chosen as the center of nuclear mass for all molecular density calculations reported here.

\section{B. Spherically averaged momentum space densities}

The spherically averaged momentum density of any molecular system can be found by expanding its wave function in a basis of one-electron momentum space functions and integrating appropriately 


$$
\pi(p)=\sum_{a b} P_{a b} \int \varphi_{a}(\mathbf{p}) \varphi_{b}(\mathbf{p}) p^{2} \sin \theta d \theta d \phi,
$$

where the momentum space basis functions $\varphi_{a}(\mathbf{p})$ are found by Dirac-Fourier transform of their position space counterparts

$$
\varphi_{a}(\mathbf{p})=(2 \pi)^{-3 / 2} \int \phi_{a}(\mathbf{r}) \exp (-i \mathbf{p} \cdot \mathbf{r}) d \mathbf{r} .
$$

If the momentum-space wave function is expanded in a basis of $s$-type momentum space Gaussian functions, the fundamental spherically averaged momentum integral can be found in closed form

$$
\begin{aligned}
{[s s]_{p}=} & (2 \alpha)^{-3 / 2}(2 \beta)^{-3 / 2} \\
& \times \int \exp \left(-\frac{p^{2}}{4 \alpha}-\frac{p^{2}}{4 \beta}-i \mathbf{p} \cdot \mathbf{R}\right) p^{2} \sin \theta d \theta d \phi, \\
= & 4 \pi p^{2}(2 \alpha)^{-3 / 2}(2 \beta)^{-3 / 2} \exp \left(-\frac{p^{2}}{4 \alpha}-\frac{p^{2}}{4 \beta}\right) j_{0}(p R),
\end{aligned}
$$

where $\mathbf{R}=\mathbf{A}-\mathbf{B}$ and $j_{0}(x)=x^{-1} \sin x$ is a spherical Bessel function of the first kind.

\section{Derivatives}

Integrals of higher angular momentum can be obtained by Boys differentiation of Eqs. (4) and (8). Following the approach of Thakkar and Sharma ${ }^{9}$ for momentum-space densities, the key to finding compact expressions for both position and momentum-space integrals lies in defining the generalized Bessel functions

$$
\begin{aligned}
& g_{n}(r P)=\frac{r^{n}}{P^{n}} i_{n}(r P), \\
& h_{n}(p R)=\frac{p^{n}}{R^{n}} j_{n}(p R)
\end{aligned}
$$

for $n \geq 0$. Using the derivative identities

$$
\begin{aligned}
& \frac{d}{d x} \frac{i_{n}(x)}{x^{n}}=\frac{i_{n+1}(x)}{x^{n}}, \\
& \frac{d}{d x} \frac{j_{n}(x)}{x^{n}}=-\frac{j_{n+1}(x)}{x^{n}},
\end{aligned}
$$

and the chain rule, it can easily be shown that

$$
\begin{aligned}
& \frac{\partial}{\partial P_{i}} g_{n}(r P)=P_{i} g_{n+1}(r P), \\
& \frac{\partial}{\partial R_{i}} h_{n}(p R)=-R_{i} h_{n+1}(p R),
\end{aligned}
$$

where $i \in\{x, y, z\}$. Application of Eq. (12a) to Eq. (10a) and Eq. (12b) to Eq. (10b) [observing that $g_{0}(r P)=i_{0}(r P)$ and $\left.h_{0}(p R)=j_{0}(p R)\right]$ leads directly to the derivatives listed in
Table I. From these derivatives, it is easy to reconstruct the required integrals. For example, the $\left[p_{x x} s\right]_{r}$ integral is

$$
\left[p_{x x} s\right]_{r}=\frac{\partial^{2}}{\partial P_{x}^{2}} g_{0}(r P)+2 A_{x} \frac{\partial}{\partial P_{x}} g_{0}(r P)+A_{x}^{2} g_{0}(r P)
$$

and its derivative components are easily found,

$$
\begin{aligned}
\frac{\partial}{\partial P_{x}} g_{0}(r P) & =P_{x} g_{1}(r P), \\
\frac{\partial^{2}}{\partial P_{x}^{2}} g_{0}(r P) & =\frac{\partial}{\partial P_{x}}\left(P_{x} g_{1}(r P)\right) \\
& =P_{x} \frac{\partial}{\partial P_{x}} g_{1}(r P)+g_{1}(r P) \frac{\partial}{\partial P_{x}} P_{x} \\
& =P_{x}^{2} g_{2}(r P)+g_{1}(r P) .
\end{aligned}
$$

For typographical simplicity, we abbreviate $P_{x}, P_{y}, P_{z}$, and $r P$ as $x, y, z$, and $s$, respectively. Likewise, $R_{x}, R_{y}, R_{z}$, and $p R$ are abbreviated as $x, y, z$, and $q$. The integrals tabulated here are sufficient for calculations with basis sets that contain up to $f$-type Gaussian basis functions.

\section{COMPUTATIONAL DETAILS}

The geometries for all molecules in the ten-electron and 18-electron isoelectronic series were taken from a previous thermochemical study. ${ }^{20}$ The one-particle density matrices required for the calculation of spherically averaged position and momentum densities were obtained using a range of $a b$ initio methods [Hartree-Fock (HF), second-order MollerPlesset perturbation theory (MP2), orbital-optimized coupled-cluster theory with double excitations (OO-CCD), and coupled-cluster theory with single and double excitations (CCSD)] in conjunction with a variety of basis sets $[3-21 \mathrm{G}, \quad 6-311 \mathrm{G}, \quad 6-311 \mathrm{G}(d, p), \quad 6-311 \mathrm{G}(3 d, 3 p)$, and $6-311 \mathrm{G}(3 d f, 3 p d)]$. All calculations, including generation of the spherically averaged integrals and contraction with the one-particle density matrix elements, were carried out within the Q-CHEM suite of quantum chemical software. ${ }^{19}$ All results are reported in atomic units.

\section{RESULTS AND DISCUSSION}

\section{A. Ten-electron series}

Benchmark [CCSD/6-311G $(3 d f, 3 p d)]$ spherically averaged position and momentum densities are presented in Fig. 1. The position densities reflect the shell structure of the heteroatoms in each molecule with a sharp, narrow peak at small $r$ (arising from core electrons in the $1 s$ orbital) and a larger, broader peak at large $r$ (arising from valence electrons in the $2 s$ and $2 p$ orbitals). Moving across the series, from neon to methane, the $1 s$ peak migrates from $0.1(\mathrm{Ne})$ through $0.25\left(\mathrm{NH}_{3}\right)$ and back to $0.15 \mathrm{Bohr}\left(\mathrm{CH}_{4}\right)$. The reason for this behavior is predominantly geometrical; the relative peak positions reflect the distance between the center of nuclear mass and the position of the heteroatom. The changes in shape and position of the valence electron peaks tell a different story, indicating that there is a monotonic transition from the relatively compact neon atom to the relatively diffuse 
TABLE I. Derivatives required for calculations of $\rho(r)$ and $\pi(p)$ using Gaussian basis functions. The order of differentiation with respect to $x, y$, and $z$ coordinates is indexed by $l, m$, and $n$, respectively. The momentum space derivatives from Ref. 9 are reproduced here for completeness (note: a small typographical error in the 420 derivative is corrected here).

\begin{tabular}{|c|c|c|c|c|c|c|c|}
\hline$l$ & $m$ & $n$ & Position space & $l$ & $m$ & $n$ & Momentum space \\
\hline 0 & 0 & 0 & $i_{0}(s)$ & 0 & 0 & 0 & $j_{0}(q)$ \\
\hline 1 & 0 & 0 & $x g_{1}(s)$ & 1 & 0 & 0 & $-x h_{1}(q)$ \\
\hline 1 & 1 & 0 & $x y g_{2}(s)$ & 1 & 1 & 0 & $x y h_{2}(q)$ \\
\hline 2 & 0 & 0 & $x^{2} g_{2}(s)+g_{1}(s)$ & 2 & 0 & 0 & $x^{2} h_{2}(q)-h_{1}(q)$ \\
\hline 3 & 0 & 0 & $x^{3} g_{3}(s)+3 x g_{2}(s)$ & 3 & 0 & 0 & $-x^{3} h_{3}(q)+3 x h_{2}(q)$ \\
\hline 2 & 1 & 0 & $x^{2} y g_{3}(s)+y g_{2}(s)$ & 2 & 1 & 0 & $-x^{2} y h_{3}(q)+y h_{2}(q)$ \\
\hline 1 & 1 & 1 & $x_{y z g_{3}}(s)$ & 1 & 1 & 1 & $-x y z h_{3}(q)$ \\
\hline 4 & 0 & 0 & $x^{4} g_{4}(s)+6 x^{2} g_{3}(s)+3 g_{2}(s)$ & 4 & 0 & 0 & $x^{4} h_{4}(q)-6 x^{2} h_{3}(q)+3 h_{2}(q)$ \\
\hline 3 & 1 & 0 & $x^{3} y_{4}(s)+3 x y g_{3}(s)$ & 3 & 1 & 0 & $x^{3} y h_{4}(q)-3 x y h_{3}(q)$ \\
\hline 2 & 2 & 0 & $x^{2} y^{2} g_{4}(s)+\left(x^{2}+y^{2}\right) g_{3}(s)+g_{2}(s)$ & 2 & 2 & 0 & $x^{2} y^{2} h_{4}(q)-\left(x^{2}+y^{2}\right) h_{3}(q)+h_{2}(q)$ \\
\hline 2 & 1 & 1 & $x^{2} y z g_{4}(s)+y z g_{3}(s)$ & 2 & 1 & 1 & $x^{2} y z h_{4}(q)-y z h_{3}(q)$ \\
\hline 5 & 0 & 0 & $x^{5} g_{5}(s)+10 x^{3} g_{4}(s)+15 x g_{3}(s)$ & 5 & 0 & 0 & $-x^{5} h_{5}(q)+10 x^{3} h_{4}(q)-15 x h_{3}(q)$ \\
\hline 4 & 1 & 0 & $x^{4} y g_{5}(s)+6 x^{2} y g_{4}(s)+3 y g_{3}(s)$ & 4 & 1 & 0 & $-x^{4} y h_{5}(q)+6 x^{2} y h_{4}(q)-3 y h_{3}(q)$ \\
\hline 3 & 2 & 0 & $x^{3} y^{2} g_{5}(s)+\left(3 x y^{2}+x^{3}\right) g_{4}(s)+3 x g_{3}(s)$ & 3 & 2 & 0 & $-x^{3} y^{2} h_{5}(q)+\left(3 x y^{2}+x^{3}\right) h_{4}(q)-3 x h_{3}(q)$ \\
\hline 3 & 1 & 1 & $x^{3} y z g_{5}(s)+3 \operatorname{xyzg}_{4}(s)$ & 3 & 1 & 1 & $-x^{3} y z h_{5}(q)+3 x y z h_{4}(q)$ \\
\hline 2 & 2 & 1 & $x^{2} y^{2} z g_{5}(s)+\left(x^{2} z+y^{2} z\right) g_{4}(s)+z g_{3}(s)$ & 2 & 2 & 1 & $-x^{2} y^{2} z h_{5}(q)+\left(x^{2} z+y^{2} z\right) h_{4}(q)-z h_{3}(q)$ \\
\hline 6 & 0 & 0 & $x^{6} g_{6}(s)+15 x^{4} g_{5}(s)+45 x^{2} g_{4}(s)+15 g_{3}(s)$ & 6 & 0 & 0 & $x^{6} h_{6}(q)-15 x^{4} h_{5}(q)+45 x^{2} h_{4}(q)-15 h_{3}(q)$ \\
\hline 5 & 1 & 0 & $x^{5} y g_{6}(s)+10 x^{3} y g_{5}(s)+15 x y g_{4}(s)$ & 5 & 1 & 0 & $x^{5} y h_{6}(q)-10 x^{3} y h_{5}(q)+15 x y h_{4}(q)$ \\
\hline 4 & 2 & 0 & $\begin{array}{l}x^{4} y^{2} g_{6}(s)+\left(6 x^{2} y^{2}+x^{4}\right) g_{5}(s) \\
+\left(6 x^{2}+3 y^{2}\right) g_{4}(s)+3 g_{3}(s)\end{array}$ & 4 & 2 & 0 & $\begin{array}{l}x^{4} y^{2} h_{6}(q)-\left(6 x^{2} y^{2}+x^{4}\right) h_{5}(q) \\
+\left(6 x^{2}+3 y^{2}\right) h_{4}(q)-3 h_{3}(q)\end{array}$ \\
\hline 4 & 1 & 1 & $x^{4} y z g_{6}(s)+6 x^{2} y z g_{5}(s)+3 y z g_{4}(s)$ & 4 & 1 & 1 & $x^{4} y z h_{6}(q)-6 x^{2} y z h_{5}(q)+3 y z h_{4}(q)$ \\
\hline 3 & 3 & 0 & $x^{3} y^{3} g_{6}(s)+\left(3 x^{3} y+3 x y^{3}\right) g_{5}(s)+9 x y g_{4}(s)$ & 3 & 3 & 0 & $x^{3} y^{3} h_{6}(q)-\left(3 x^{3} y+3 x y^{3}\right) h_{5}(q)+9 x y h_{4}(q)$ \\
\hline 3 & 2 & 1 & $x^{3} y^{2} z g_{6}(s)+\left(x^{3} z+3 x y^{2} z\right) g_{5}(s)+3 x z g_{4}(s)$ & 3 & 2 & 1 & $x^{3} y^{2} z h_{6}(q)-\left(x^{3} z+3 x y^{2} z\right) h_{5}(q)+3 x z h_{4}(q)$ \\
\hline 2 & 2 & 2 & $\begin{array}{l}x^{2} y^{2} z^{2} g_{6}(s)+\left(x^{2} y^{2}+x^{2} z^{2}+y^{2} z^{2}\right) g_{5}(s) \\
+\left(x^{2}+y^{2}+z^{2}\right) g_{4}(s)+g_{3}(s)\end{array}$ & 2 & 2 & 2 & $\begin{array}{l}x^{2} y^{2} z^{2} h_{6}(q)-\left(x^{2} y^{2}+x^{2} z^{2}+y^{2} z^{2}\right) h_{5}(q) \\
+\left(x^{2}+y^{2}+z^{2}\right) h_{4}(q)-h_{3}(q)\end{array}$ \\
\hline
\end{tabular}

methane molecule. In this case, the geometrical effects that strongly influence the location of the $1 s$ peak pale into insignificance beside the chemical changes in the behavior of the valence electrons.
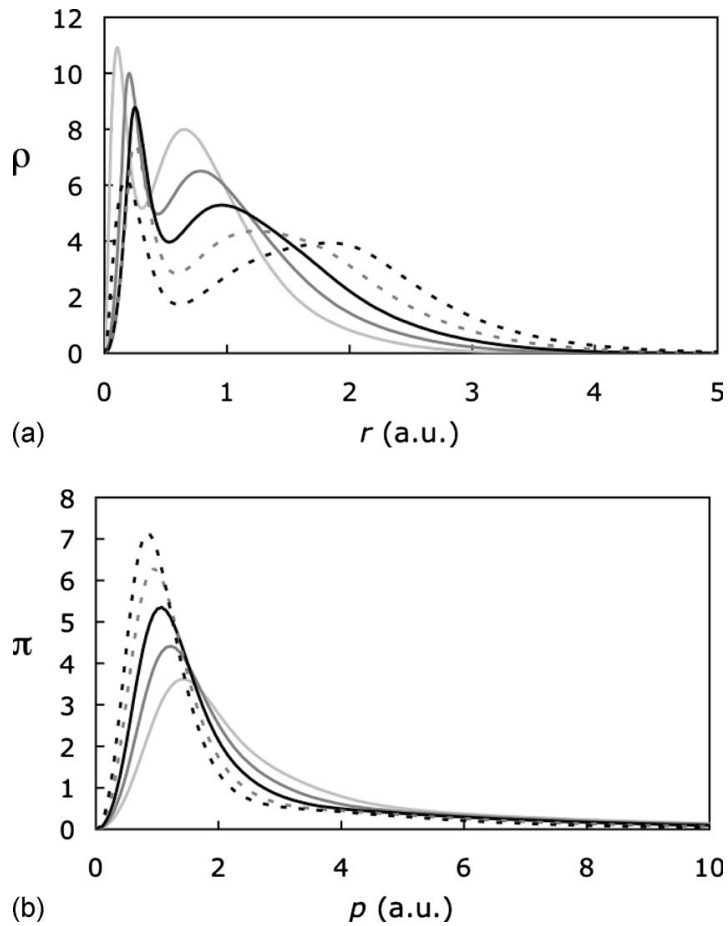

FIG. 1. Spherically averaged (a) position and (b) momentum densities for the ten-electron series $\left(\mathrm{Ne}=\right.$ light gray line, $\mathrm{HF}=$ dark gray line, $\mathrm{H}_{2} \mathrm{O}$ = black line, $\mathrm{NH}_{3}=$ dashed gray line, and $\mathrm{CH}_{4}=$ dashed black line).
By contrast, the momentum densities are relatively uninteresting, featuring a single peak in the $p=1-2 \mathrm{Bohr}^{-1}$ range. Nonetheless, they provide a useful complementary perspective on the changes in electron behavior that occur moving across the isoelectronic series. The most compact system, neon, has the fastest-moving electrons, and there is a monotonic transition to the most diffuse system, methane, whose electrons move the slowest. This complementarity in the position and momentum-space pictures of electron behavior has also been neatly illustrated in previous work by Thakkar $^{14}$ using the beryllium atom as an example. In this case, the $1 s$ electrons, which are closest to the nucleus and moving fastest, show up as a short-range peak in $\rho(r)$ and a long-range peak in $\pi(p)$. Conversely, the $2 s$ electrons are responsible for a long-range peak in $\rho(r)$ and a short-range peak in $\pi(p)$.

\section{Binding densities}

A large component of both the position and momentum space densities is geometrical in nature, arising from the fact that a molecule is basically a collection of near-spherical atoms situated at different points in space. However, chemistry, as a discipline, is concerned with precisely this distinction quantifying the difference between exactly spherical noninteracting atoms and the changes that occur as a result of bonding interactions. To directly probe this effect, it is helpful to define position $(r)$ and momentum $(p)$ space interaction difference densities, ${ }^{21}$ 


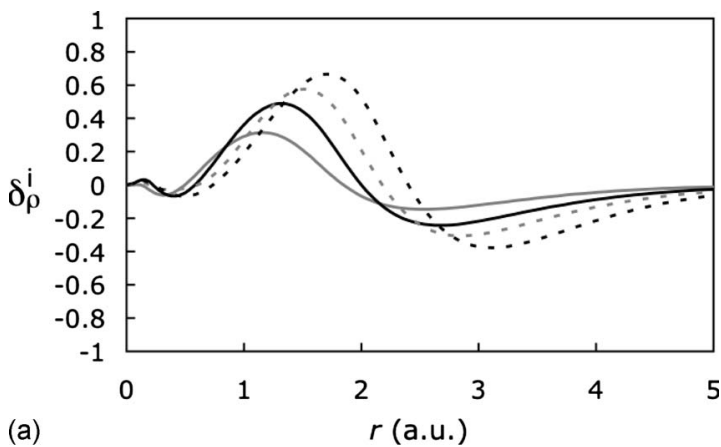

(a)

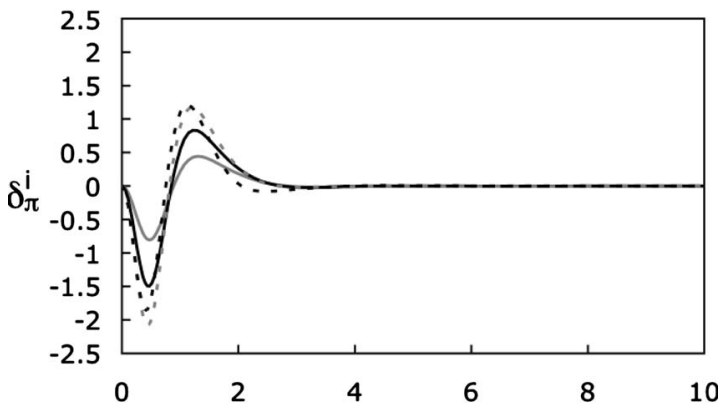

(b)

p (a.u.)

FIG. 2. (a) Position and (b) momentum interaction difference densities for the ten-electron series $\left(\mathrm{HF}=\right.$ dark gray line, $\mathrm{H}_{2} \mathrm{O}=$ black line, $\mathrm{NH}_{3}=$ dashed gray line, and $\mathrm{CH}_{4}=$ dashed black line).

$$
\begin{aligned}
& \delta_{\rho}^{i}(r)=\rho(r)-\sum_{j=0}^{\text {natom }} \rho_{j}(r), \\
& \delta_{\pi}^{i}(p)=\pi(p)-\sum_{j=0}^{\text {natom }} \pi_{j}(p),
\end{aligned}
$$

where $\rho_{j}(r)$ and $\pi_{j}(p)$ are atomic position and momentum densities.

The interaction difference densities for all molecules in the ten-electron series are illustrated in Fig. 2. From this figure, it is easy to see that a very little change to the core electrons occurs upon binding in either the short-range $(0-0.5$ Bohr $)$ section of $\rho(r)$ or the long-range $\left(>2.5 \mathrm{Bohr}^{-1}\right.$ ) region of $\pi(p)$. The $\delta_{\rho}^{i}(r)$ show a buildup of charge density in the bonding region (1-2 Bohr) and a depletion at long range. The $\delta_{\pi}^{i}(p)$, on the other hand, indicate an increase in the average momentum of the valence electrons. This is consistent with the $r$-space picture, as bonding electrons are expected to move faster than electrons at long range. Finally, we note that the maximum magnitude of $\delta_{\rho}^{i}(r)$ and $\delta_{\pi}^{i}(p)$ increases along the ten-electron series. Neon, by definition, has a zero interaction density for all values of $r$ and $p$. Methane, with the largest number of bonds, has the most pronounced interaction density in position space. The $p$-space interaction densities of $\mathrm{NH}_{3}$ and $\mathrm{CH}_{4}$ are similar, although the absolute magnitude of the ammonia interaction density is slightly larger.

\section{Basis set and correlation effects}

Although CCSD/6-311G $(3 d f, 3 p d)$ is expected to yield excellent benchmark densities, ${ }^{15,22-27}$ it is important to check

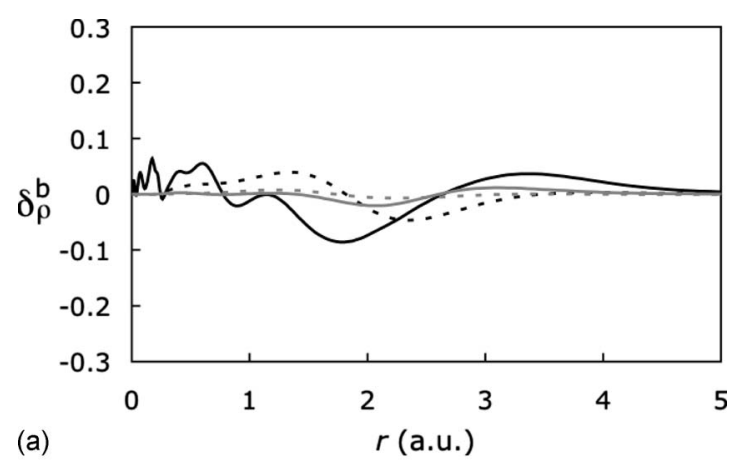

(a)

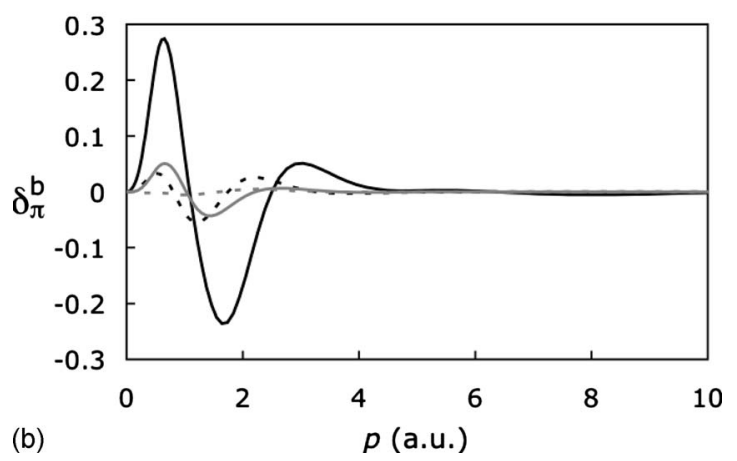

FIG. 3. (a) Position and (b) momentum basis difference densities for the ten-electron series averaged over all molecules $(3-21 \mathrm{G}=$ black line, $6-311 \mathrm{G}=$ grey line, 6-311G $(d, p)=$ dashed black line, and $6-311 \mathrm{G}(3 d, 3 p)$ $=$ dashed gray line).

convergence with respect to both basis set size and treatment of electron correlation. Basis set and correlation difference densities are defined as

$$
\begin{aligned}
& \delta_{\rho}^{b}(r)=\rho^{\mathrm{CCSD} / \operatorname{large}}(r)-\rho^{\mathrm{CCSD} / X}(r), \\
& \delta_{\pi}^{b}(p)=\pi^{\mathrm{CCSD} / \operatorname{large}(}(p)-\pi^{\mathrm{CCSD} / X}(p), \\
& \delta_{\rho}^{c}(r)=\rho^{\mathrm{CCSD} / \operatorname{large}(}(r)-\rho^{Z / \operatorname{large}(r),} \\
& \delta_{\pi}^{c}(p)=\pi^{\mathrm{CCSD} / \operatorname{large}(p)-\pi^{Z / \operatorname{large}}(p),}
\end{aligned}
$$

where

large $=6-311 \mathrm{G}(3 d f, 3 p d)$,

$X \in\{3-21 \mathrm{G}, 6-311 \mathrm{G}, 6-311 \mathrm{G}(d, p), 6-311 \mathrm{G}(3 d, 3 p)\}$

and $Z \in\{\mathrm{HF}, \mathrm{MP} 2, \mathrm{OO}-\mathrm{CCD}\}$.

Basis set difference densities for all molecules in the ten-electron series were found to be qualitatively and quantitatively similar. The difference densities presented in Fig. 3 were therefore generated by averaging over the entire data set. Of all the basis sets, only 3-21G performs exceptionally poorly, giving errors that are either erratic ( $r$-space) or substantial relative to the interaction difference density ( $p$-space). The 6-311G and 6-311G $(d, p)$ basis sets differ from the benchmark $6-311 \mathrm{G}(3 d f, 3 p d)$ result by at most 0.05 electrons $\left(e^{-}\right)$, and their error profiles are smooth. For most purposes, these basis sets would be sufficiently accurate. However, inclusion of extra polarization functions significantly improves the performance of the basis, and the 


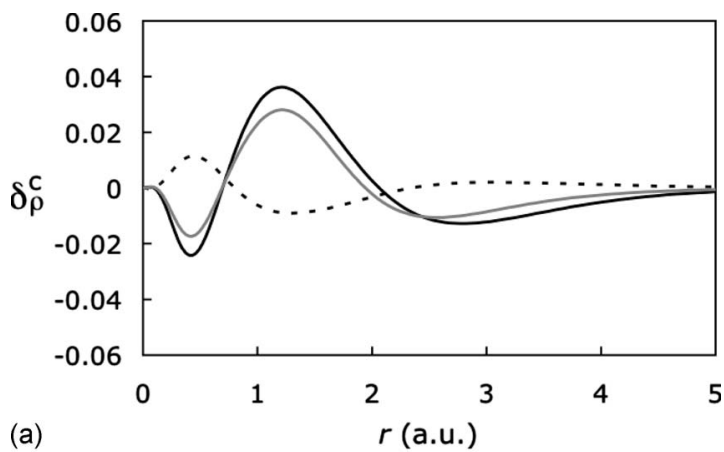

(a)

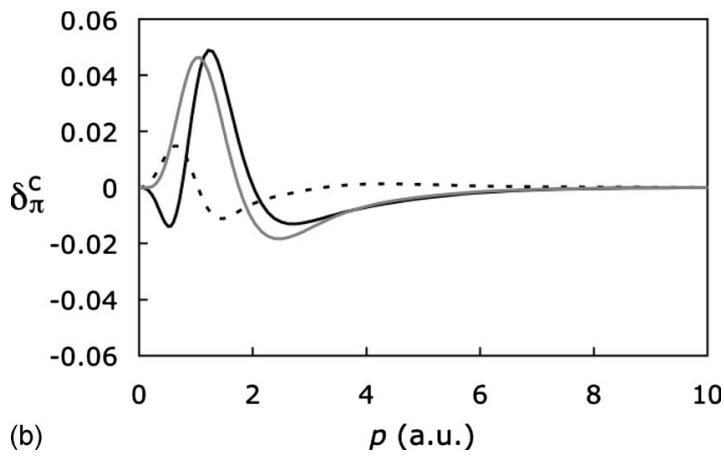

FIG. 4. (a) Position and (b) momentum correlation difference densities for the ten-electron series averaged over all molecules $($ Hartree-Fock $=$ dashed black line, MP2 = grey line, and $\mathrm{OO}-\mathrm{CCD}=$ black line).

6-311G $(3 d, 3 p)$ data are almost identical to the benchmark $6-311 \mathrm{G}(3 d f, 3 p d)$ results. This is consistent with previous literature results, ${ }^{12}$ which indicate that the pc-1 basis set, which is of a similar quality to $6-311 \mathrm{G}(d, p)$, is among the smallest that is capable of reproducing benchmark results to within $0.5 \%$ for most values of $r$ and $p$. Larger basis sets are required if an accurate description of very short-range or very long-range behavior is required.

Correlation difference densities for all molecules in the ten-electron series were also found to be similar and averaged difference densities are presented in Fig. 4. From this figure, two conclusions are immediately obvious. First, correlated densities tend to slightly enhance the probability of finding electrons at small values of $r$ and $p$, relative to a HF reference. Second, MP2 and OO-CCD both tend to overestimate the effect of correlation relative to the benchmark CCSD results. Both of these conclusions are in agreement with previous studies on similar systems. ${ }^{15-17,24}$ However, in all cases, the magnitude of $\delta_{\rho}^{c}$ and $\delta_{\pi}^{c}$ is small, which suggests that the density is not very sensitive to the treatment of electron correlation. This is also supported by previous studies, ${ }^{10,17,28}$ which confirm that correlation difference densities tend to be over an order of magnitude smaller than interaction difference densities.

Therefore, for most typical organic molecules, the HF density will be a sufficiently accurate approximation to the true density. For the molecules investigated here, HF/6-311G provides an optimal balance between performance and computational expense, assuming a $0.05 e^{-}$tolerance. However, this conclusion may not hold for all systems. For example, it is likely that negatively charged ions would require a basis set that includes diffuse functions, and a more sophisticated treatment of electron correlation may be required for strongly multireference systems such as diradicals and unsaturated molecules. In this case, it would be interesting to investigate the possibility of adding a scaled MP2 correlation correction to the HF density, given the systematic exaggeration of the correlation effect by MP2.

\section{B. Eighteen-electron series}

Spherically averaged densities and interaction difference densities for the 18-electron series are presented in Figs. 5 and 6, respectively. From Fig. 5, it can be seen that the $\mathrm{Ar}-\mathrm{SiH}_{4}$ isoelectronic series broadly follows the same trends as the $\mathrm{Ne}-\mathrm{CH}_{4}$ series, although extra shell structure is apparent in both $r$ - and $p$-spaces. While the $\mathrm{F}_{2}-\mathrm{H}_{3} \mathrm{CCH}_{3}$ momentum densities are very similar to their $\mathrm{HF}-\mathrm{CH}_{4}$ counterparts, their position densities are strikingly different. Again, this is a predominantly geometrical effect, as the peak locations reflect the distance between the heavy atoms in each molecule and the center of mass. Therefore, to understand what is really happening in terms of bonding in these systems, it is necessary to turn to the interaction difference densities presented in Fig. 6.

The $\mathrm{Ar}-\mathrm{SiH}_{4} \delta_{\rho}^{i}(r)$ and $\delta_{\pi}^{i}(p)$ are very similar to their first-row counterparts, albeit with an extended core region that encompasses both the $1 s$ and $2 s / 2 p$ shells. Likewise, the $\mathrm{F}_{2}-\mathrm{H}_{3} \mathrm{CCH}_{3} p$-space interaction densities are very similar to their $\mathrm{HF}-\mathrm{CH}_{4}$ counterparts, although their $r$-space interaction densities are highly dissimilar. To interpret the behavior of $\delta_{\rho}^{i}(r)$, it is necessary to bear in mind that the distance between the center of mass and the heavy atoms of each molecule is around 1.2 Bohr. Therefore, any positive interaction density inside this region is associated with the heteroatom-heteroatom bond while any positive interaction density outside this region is augmenting the heteroatomhydrogen bonds. According to this analysis, all of the polyatomic molecules behave as expected, withdrawing electron density from long-range regions to augment the charge density around the bonding regions of the system. $F_{2}$ is an anomaly in this respect, as it redistributes electrons away from the interatomic region relative to a protomolecule comprised of two noninteracting atoms.

\section{CONCLUSIONS}

The work presented in this paper describes the derivation of new, compact expressions for spherically averaged position density integrals based on a Gaussian-type orbital wave function expansion. All integrals needed for spherically averaged position and momentum density calculations involving $s, p, d$, and $f$-type Gaussians are tabulated, highlighting a neat isomorphism between the position and momentum space formulae. Benchmark position and momentum densities were calculated based on CCSD/6-311G(3df,3pd) wave functions, and the bonding-induced component of these densities was analyzed by comparison with $\mathrm{CCSD} / 6-311 \mathrm{G}(3 d f, 3 p d)$ protomolecule densities. Extensive testing of basis set and correlation effects on spherically averaged densities revealed that for most purposes, HF/6$311 \mathrm{G}$ densities are sufficiently accurate with a maximum 

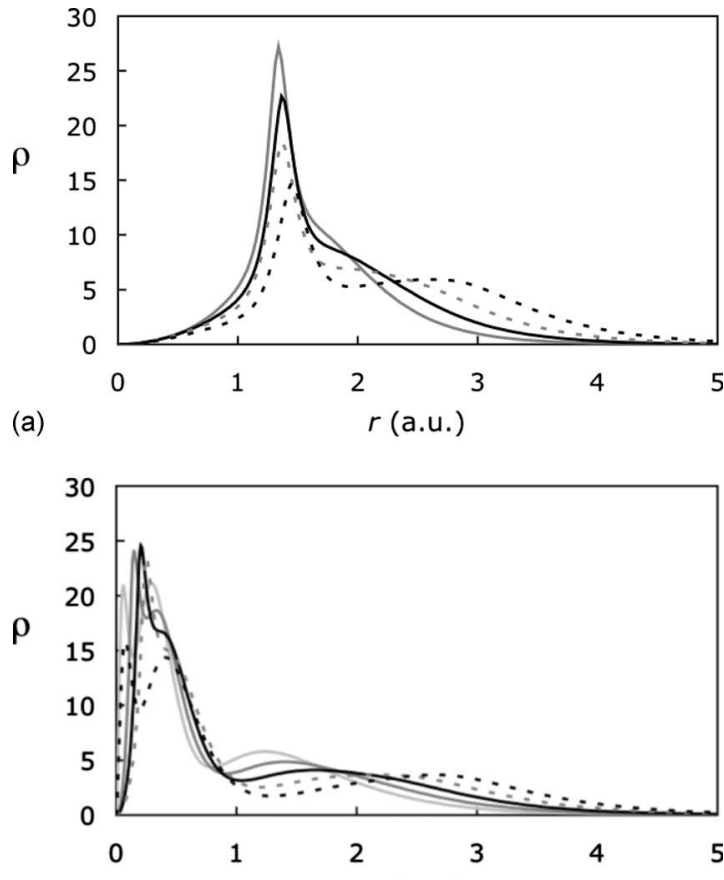

(b) $r$ (a.u.)
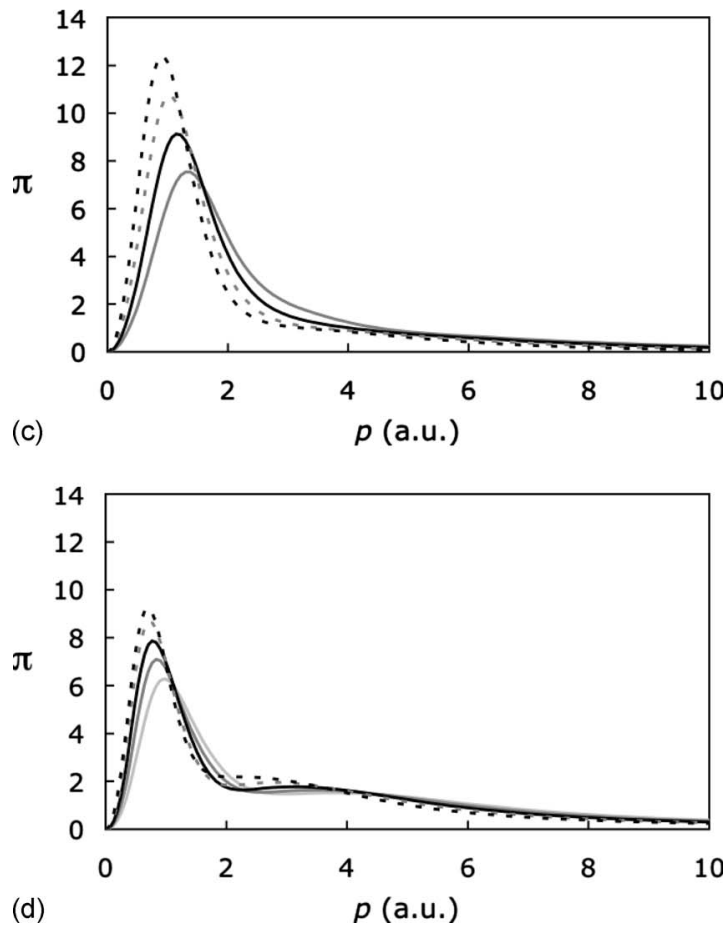

FIG. 5. Spherically averaged [(a) and (b)] position and [(c) and (d)] momentum densities for the 18-electron series: [(a) and (c)] $\mathrm{Ar}=$ light gray line, $\mathrm{HCl}=$ dark gray line, $\mathrm{H}_{2} \mathrm{~S}=$ black line, $\mathrm{PH}_{3}=$ dashed gray line, and $\mathrm{SiH}_{4}$ $=$ dashed black line; $[(\mathrm{b})$ and (d) $] \mathrm{F}_{2}=$ dark gray line, $\mathrm{HOOH}=$ black line, $\mathrm{H}_{2} \mathrm{NNH}_{2}$ =dashed gray line, and $\mathrm{H}_{3} \mathrm{CCH}_{3}=$ dashed black line.

relative error in the density of $0.5 \%$. The systematic overestimation of the correlation effect by MP2 suggests that using a scaled MP2 correlation correction may provide a costeffective method of estimating the true correlated density.

\section{ACKNOWLEDGMENTS}

A generous allocation of computer time from the Australian National Computational Infrastructure National Facility

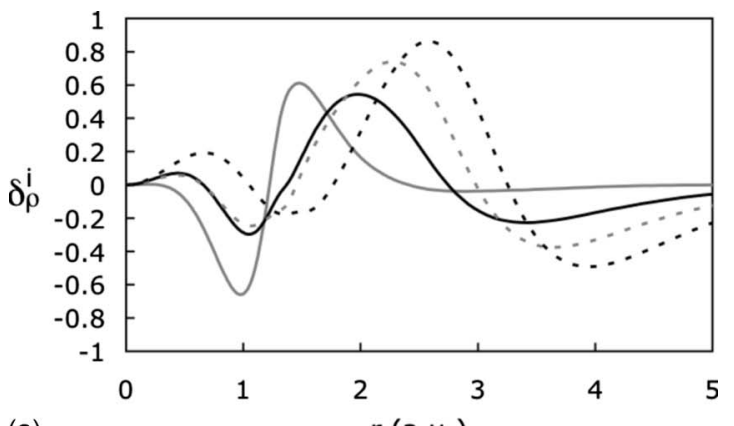

(a)

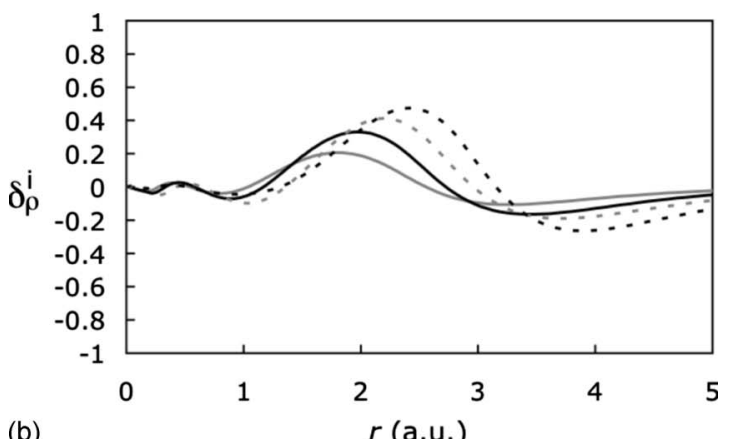

(b)
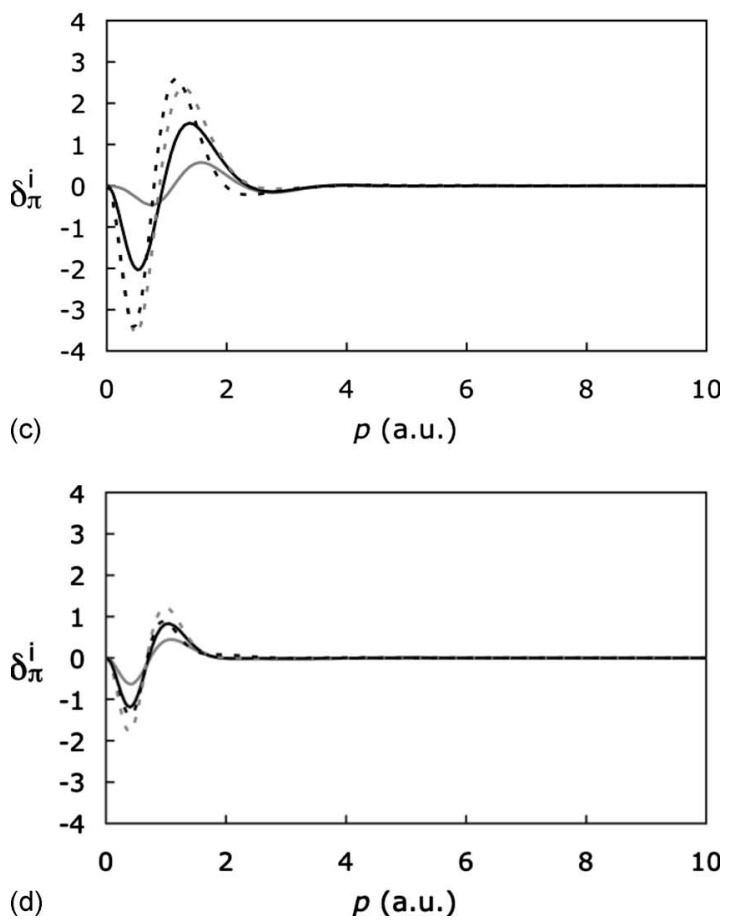

FIG. 6. [(a) and (b)] Position and [(c) and (d)] momentum interaction difference densities for the 18-electron series: [(a) and (c) $] \mathrm{HCl}=$ dark gray line, $\mathrm{H}_{2} \mathrm{~S}$ =black line, $\mathrm{PH}_{3}=$ dashed gray line, and $\mathrm{SiH}_{4}=$ dashed black line; [(b) and (d) $] \mathrm{F}_{2}=$ dark gray line, $\mathrm{HOOH}=$ black line, $\mathrm{H}_{2} \mathrm{NNH}_{2}=$ dashed gray line, and $\mathrm{H}_{3} \mathrm{CCH}_{3}=$ dashed black line.

is gratefully acknowledged. This work was also supported by an Australian Research Council grant to Professor Peter Gill (Grant No. DP0664466), and the authors thank PMWG for helpful feedback and discussions.

${ }^{1}$ J. H. V. Vleck, The Theory of Electric and Magnetic Susceptibilities (Oxford University Press, New York, 1932).

${ }^{2} \mathrm{D}$. W. Davies, The Theory of the Electric and Magnetic Properties of Molecules (Wiley, New York, 1966). 
${ }^{3}$ J. Wang and V. H. Smith, Jr., J. Phys. B 27, 5159 (1994).

${ }^{4}$ R. A. Bonham, J. Phys. Chem. 27, 856 (1994).

${ }^{5}$ I. R. Epstein, Phys. Rev. A 27, 160 (1994).

${ }^{6}$ P. Kaijser and V. H. Smith, Jr., Adv. Quantum Chem. 10, 37 (1977).

${ }^{7}$ J. Wang and V. H. Smith, Jr., Int. J. Quantum Chem. 52, 1145 (1994).

${ }^{8}$ C. Sarasola, J. M. Elorza, and J. M. Ugalde, J. Math. Chem. 23, 405 (1998).

${ }^{9}$ A. J. Thakkar and B. S. Sharma, Int. J. Quantum Chem. 85, 258 (2001).

${ }^{10}$ H. Meyer, T. Muller, and A. Schweig, J. Mol. Struct.: THEOCHEM 360, 55 (1996).

${ }^{11}$ S. K. Worsnop, R. J. Boyd, J. M. Elorza, C. Sarasola, and J. M. Ugalde, J. Chem. Phys. 112, 1113 (2000).

${ }^{12}$ J. R. Hart and A. J. Thakkar, Int. J. Quantum Chem. 102, 673 (2005).

${ }^{13}$ B. Miguel and J. M. G. de la Vega, Theor. Chem. Acc. 118, 723 (2007).

${ }^{14}$ A. J. Thakkar, Adv. Chem. Phys. 128, 303 (2004).

${ }^{15}$ F. de Proft and P. Geerlings, Chem. Phys. Lett. 220, 405 (1994).

${ }^{16}$ V. H. Smith, Jr., Phys. Scr. 15, 147 (1977).

${ }^{17}$ V. H. Smith, Jr. and I. Absar, Isr. J. Chem. 16, 87 (1977).

${ }^{18}$ P. Kaijser, V. H. Smith, Jr., A. N. Tripathi, and G. H. F. Diercksen, Phys. Rev. A 35, 4074 (1987).

${ }^{19}$ Y. Shao, L. Fusti-Molnar, Y. Jung, J. Kussmann, C. Ochsenfeld, S. T. Brown, A. T. B. Gilbert, L. V. Slipchenko, S. V. Levchenko, D. P. O’Neill, R. A. DiStasio, Jr., R. C. Lochan, T. Wang, G. J. O. Beran, N. A.
Besley, J. M. Herbert, C. Y. Lin, T. Van Voorhis, S. Hung Chien, A. Sodt, R. P. Steele, V. A. Rassolov, P. E. Maslen, P. P. Korambath, R. D. Adamson, B. Austin, J. Baker, E. F. C. Byrd, H. Dachsel, R. J. Doerksen, A. Dreuw, B. D. Dunietz, A. D. Dutoi, T. R. Furlani, S. R. Gwaltney, A. Heyden, S. Hirata, C.-P. Hsu, G. Kedziora, R. Z. Khalliulin, P. Klunzinger, A. M. Lee, M. S. Lee, W. Liang, I. Lotan, N. Nair, B. Peters, E. I. Proynov, P. A. Pieniazek, Y. Min Rhee, J. Ritchie, E. Rosta, C. David Sherrill, A. C. Simmonett, J. E. Subotnik, H. Lee Woodcock III, W. Zhang, A. T. Bell, and A. K. Chakraborty, Phys. Chem. Chem. Phys. 8, 3172 (2006).

${ }^{20}$ D. P. O'Neill and P. M. W. Gill, Mol. Phys. 103, 763 (2005).

${ }^{21}$ F. L. Hirshfeld and S. Rzotkiewicz, Mol. Phys. 27, 1319 (1974).

${ }^{22}$ D. C. Rawlings and E. R. Davidson, J. Phys. Chem. 89, 969 (1985).

${ }^{23}$ J. Blomquist and H. Essen, Chem. Phys. 112, 173 (1987).

${ }^{24}$ K. B. Wiberg, C. M. Hadad, T. J. LePage, C. M. Breneman, and M. J. Frisch, J. Phys. Chem. 96, 671 (1992).

${ }^{25}$ R. Kobayashi, H. Koch, P. Jorgensen, and T. J. Lee, Chem. Phys. Lett. 211, 94 (1993).

${ }^{26}$ J. L. Stuber and J. Paldus, J. Mol. Struct.: THEOCHEM 591, 219 (2002).

${ }^{27}$ A. D. Bochevarov and R. A. Friesner, J. Chem. Phys. 128, 034102 (2008).

${ }^{28}$ G. Das and A. C. Wahl, J. Chem. Phys. 44, 87 (1966). 